\title{
UCRL-TR-212369
}

LAW RENCE LIVERMORE N A TIO N A L LABORATORY

\section{The Need for a Neutron Source at the Rare Isotope Accelerator}

L. E. Ahle, B. Rusnak, K. E. Roberts, M. D. Roeben, M. Hausmann, R. Reifarth, D. Vieira

May 18, 2005 
This document was prepared as an account of work sponsored by an agency of the United States Government. Neither the United States Government nor the University of California nor any of their employees, makes any warranty, express or implied, or assumes any legal liability or responsibility for the accuracy, completeness, or usefulness of any information, apparatus, product, or process disclosed, or represents that its use would not infringe privately owned rights. Reference herein to any specific commercial product, process, or service by trade name, trademark, manufacturer, or otherwise, does not necessarily constitute or imply its endorsement, recommendation, or favoring by the United States Government or the University of California. The views and opinions of authors expressed herein do not necessarily state or reflect those of the United States Government or the University of California, and shall not be used for advertising or product endorsement purposes.

This work was performed under the auspices of the U.S. Department of Energy by University of California, Lawrence Livermore National Laboratory under Contract W-7405-Eng-48. 


\title{
The Need for a Neutron Source at the Rare Isotope Accelerator
}

\author{
Larry Ahle, Brian Rusnak, Kevin Roberts, Marty Roeben \\ Lawrence Livermore National Laboratory
}

\author{
Marc Hausmann, Rene Reifarth, Dave Vieira \\ Los Alamos National Laboratory
}

January 10, 2005

This work was performed under the auspices of the U.S. Department of Energy by the University of California, Lawrence Livermore National Laboratory under contract No. W-7405-Eng-48 and by Los Alamos National Laboratory under contract No. W-7045-ENG-36. 


\section{Executive Summary}

An intense neutron source facility with radiochemical processing capability is necessary at the Rare Isotope Accelerator (RIA) to fully realize its potential benefit to stockpile stewardship and astrophysics. Both stockpile steward and astrophysics require neutron cross-section information on unstable nuclei and the unprecedented production rates expected at RIA enable many of these measurements but only if the proper capabilities are present. Specifically, it must be possible to collect the desired isotopes, transport that sample to a radiochemical facilities for processing into an irradiation target, and position the target in front of an intense neutron flux of the appropriate energy. And all of this must be done before the before the sample decays away. Since the half-life of the sample may be as short as one day and the activity as high as 10 Curies, it is only practical to do these measurements on the RIA site.

Given the expected production rates at RIA, 10 Curies of a given isotope is the most that can be collected, but other radioactive isotopes may be present in the collected sample. Thus, the radiochemistry areas must be able to handle 100 Curies of hard gamma-ray activity. Also, in order to meet the requirements of the astrophysics and stockpile stewardship communities, an intense, "monoenergetic", energy tunable neutron source is needed. The range of energy of interest is from $5 \mathrm{keV}$ to $17 \mathrm{MeV}$, though no single reaction requires that entire energy range. There is a large variance in the required neutron flux due to the large variance in nuclear and chemical properties of the nuclei in question. Based on the expected production rates at RIA, some measurement can be done with a flux as low as $10^{6} \mathrm{n} / \mathrm{cm}^{2} / \mathrm{sec}$ while others require fluxes of $10^{10} \mathrm{n} / \mathrm{cm}^{2} / \mathrm{sec}$ or greater. There is also a need to accommodate several different experimental techniques, from the simple activation method, to more advanced prompt techniques that detect the reactions as they occur. These prompt techniques usually require some sort of detector array such as the DANCE detector at the LANSCE facility at Los Alamos.

A first conceptual design of a facility meeting the above requirements has been completed and the results are presented here. The radiochemistry area has three different hot cells each capable of handling 100 Curies of hard gamma ray activity. There are two different accelerators to meet the energy, flux, and timing structure requirements of the neutron cross section measurements. There are also three different experimental areas or neutron caves because of the differing challenges faced with the different neutron cross-section measurements. A preliminary cost analysis has performed and its results are summarized at the table to the right.

\begin{tabular}{|l|c|}
\hline Cost Summary & Cost $(\$ \mathrm{M})$ \\
\hline Building & 17.0 \\
\hline Accelerators & 36.2 \\
\hline Radiochemistry Area & 24.5 \\
\hline Experimental Equipment & 22.7 \\
\hline TOTAL & 100.4 \\
\hline
\end{tabular}

The Rare Isotope Accelerator presents the best and only opportunity to measure many neutron cross sections important to stockpile stewardship and astrophysics. Fully realizing its potential will require an onsite neutron source with radiochemical capabilities. Such a facility should be technically feasible and should have minimal impact on the rest of the RIA facility. 


\section{Introduction}

An intense neutron source facility with radiochemical processing capability is necessary at the Rare Isotope Accelerator to fully realize its potential benefit to stockpile stewardship and astrophysics. While many of the important physics missions of RIA can be addressed with radioactive ion beams, direct neutron cross-section measurements of interest to stockpile stewardship and astrophysics cannot because one cannot make a neutron target. Thus, one must collect a sufficient amount of the appropriate short-lived isotope, quickly chemically process the material into a target, and promptly radiate the sample with an intense "beam" of neutrons. The unprecedented production rates expected at RIA enables many of these direct neutron cross-section measurements, but only if the proper infrastructure is in place. This document not only describes the major piece of this required infrastructure, a neutron source facility with radiochemical processing capabilities, but also the motivation for measuring such direct neutron crosssections.

\section{The Need for Direct Neutron Cross-Section Measurements on Short-Lived Nuclei}

To understanding nuclear weapon test data and the origin of the elements heavier than iron a detailed knowledge of neutron cross-section on a number of near-stability nuclei is required. For astrophysics, $(\mathrm{n}, \gamma)$ cross-sections on neutron-rich nuclei are required, while for stockpile stewardship $(n, \gamma)$ and $(n, 2 n)$ cross-sections for proton-rich nuclei are the most important neutron reactions. While the motivations are different, both scientific endeavors involve understanding what happens to nuclei in intense neutron flux environments.

\subsection{Stockpile Stewardship}

\begin{tabular}{|l|l|l|l|}
\hline Reaction & $\begin{array}{l}\text { Energy Range } \\
(\mathbf{M e V})\end{array}$ & Importance & $\begin{array}{l}\text { Desired } \\
\text { Accuracy }\end{array}$ \\
\hline$(\mathrm{n}, \gamma)$ & $0.01-.0 .2$ & High & $10 \%$ \\
\hline$(\mathrm{n}, \mathrm{n})$ & $1-10$ & Low & $10 \%$ \\
\hline$(\mathrm{n}, 2 \mathrm{n})$ & $10-16$ & High & $3-5 \%$ \\
\hline$(\mathrm{n}, \alpha),(\mathrm{n}, \mathrm{pxn})$ & $0.1-16$ & Medium & $10 \%$ \\
\hline$(\mathrm{n}, \mathrm{f})$ & $0.1-16$ & High & $1-2 \%$ \\
\hline
\end{tabular}

Table 1: Neutron Reactions Important to Stockpile Stewardship

In order to obtain information about the neutron flux during a nuclear weapons test, certain isotopes were used as flux monitors. The intense neutron flux of the test would initiate nuclear reactions on these monitors, creating radioactive nuclei that could be collected and measured after the test. Given the detection of these produced nuclei, it is possible to infer information about the neutron flux of the event, provided accurate cross 
section information is known not only for the loaded stable isotope, but for several nearby radioactive isotopes as well. Some of the nuclear reactions that take place in these tests are $(n, 2 n),(n, \gamma),\left(n, n^{\prime}\right),(n, p),(n, \alpha)$, and $(n, n p)$, with $(n, 2 n)$ and $(n, \gamma)$ being in general the more important reactions. But given the difficulty in performing neutron crosssection measurement on radioactive targets, most of the cross-sections used are determined from theory alone. Thus, a major effort in reducing the uncertainty of simulations of the nuclear explosions is to reduce the uncertainty into the nuclear data used as input for the models. Obtaining new data for neutron cross-section on radioactive nuclei is one way to do this. Table 1 lists the various reactions important to stockpile stewardship, the relevant range of neutron energy, and the desired measurement accuracy.

\subsection{Astrophysics}

Stars with mass less than about 8 solar mass units will enter a stellar phase during which high neutron fluxes exist in the intermediate helium shell of the star. This enables a nucleosynthesis process known as the s-process, or slow neutron capture process. In the s-process, seed nuclei experience successive neutron captures until an unstable isotope is reached which undergoes beta-decays forming the next heavier element. The process is repeated many times to form the elements between iron and bismuth. In most cases the nuclei either live long or short compared to the mean neutron capture time making determination of the next step of the process simple, either a neutron capture or beta decay, respectively. When these times are comparable, then accurate data of the neutron capture cross-section is required in order to understand in detail the effect the s-process has on the observed abundances of the various elements. Thus, one of the major goals of nuclear astrophysics is to precisely measure the neutron capture cross-sections for these branch point nuclei for neutron energies from 1-100 keV.

\section{Present Limitations and Promise of RIA}

The biggest challenge in performing radioactive target neutron cross-section experiments is getting enough material for a target. This is exasperated by the fact neutron "beams" are much less intense than charged particle beams and the time for neutron irradiation is limited by the lifetime of the radioactive target. Most of the "monoenergetic" techniques for making neutrons are limited by beam heating in the production target. RTNS delivered a source term of $6 \times 10^{12}$ neutrons/second but only with much engineering effort on a rotating tritium target. Yields of $10^{9-11} \mathrm{n} / \mathrm{s}$ are more typical for intense neutron sources, with many present day "monoenergetic" sources operating well below that level. Given the need to be at least some distance away from the source, fluxes of $10^{10}$ $\mathrm{n} / \mathrm{cm}^{2} / \mathrm{sec}$ are then a reasonable upper limit as to what can be achieved. Assuming a cross-section of $100 \mathrm{mb}$ for the reaction of interest and a 10 day irradiation, then only $1 \mathrm{in}$ $10^{9}$ of the target atoms will undergo the desired reaction. Further assuming $1 \%$ detection efficiency and the need to detect 1000 events (3\% statistical error) than a target of $10^{15}$ atoms is the absolute minimum. If the half-life of the target material is 10 days and one collects the target material for 10 days then a production rate of $4 \times 10^{9}$ particles/second is required. One should note that the numbers used above only provide an estimate within one to two orders of magnitude. There can be significant differences in the assumptions (cross-sections, neutron flux, detection efficiency) above depending on the specific nuclei of interest and the type measurement being performed. Of course, the half-life of the 
proposed target will have the largest impact on the required production rate, due to limits in collection and irradiation time.

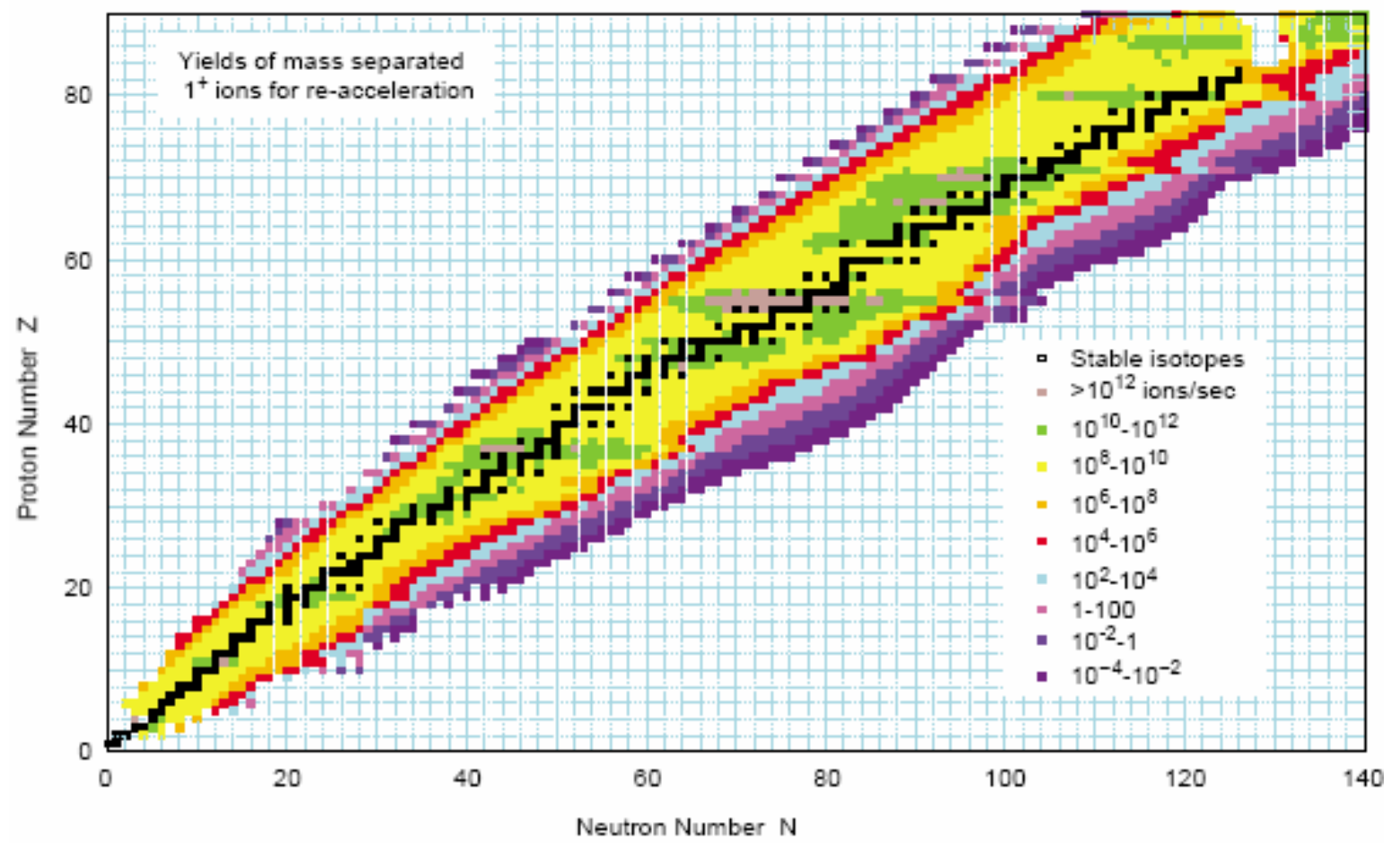

Figure 1: Expected Production Rates at RIA

One way presently available to make radioactive isotopes is to use light ion reactions, such as $(p, n)$ or $(\alpha, 2 n)$, on thick targets of nearby stable nuclei at low energies $(\sim 10-20$ $\mathrm{MeV})$. This technique however, is only applicable for very near stability, proton-rich, light mass nuclei. The low energy of the beam prohibits the making of isotopes even moderately far from stability and the removal of neutrons limits one to proton rich nuclei. (The cross-sections for charge-particle out reactions are much reduced by the Coulomb barrier). Furthermore, as one moves to heavier and heavier nuclei, the Coulomb barrier limits these light ion reactions, as the desired beam energy is no longer above the Coulomb barrier. A further complication to this approach is obtaining the required purity of the desired nuclei. Only chemical separation is readily available with this technique, resulting in the need to use isotopically pure production targets and low beam energies to reduce the number of available reaction channels. These limits imply that this method is impractical for most nuclei of interest to stockpile stewardship and astrophysics.

Reactors offer another method for producing radioactive material. The high flux of low energy neutrons will create radioactive isotopes through neutron capture events. As such it is not a good method for creating nuclei relevant to stockpile stewardship which are predominantly proton rich. The s-process branch points are neutron rich and some can be made in reactors. However, RIA also offers the capability of mass separation which is not easily accomplished if the nuclei are produced at a reactor. 
Figure 1 shows the expected production rates of RIA across the entire table of isotopes [1]. The chart clearly shows that for many near stability nuclei RIA will provide mass separated production rates of $10^{10-12}$ particles/per second and will reach as far as 10 or more nucleons from stability. This is at least of a factor of $4-5$, and often several orders of magnitude, more than any other existing or planned radioactive ion beam facility [2].

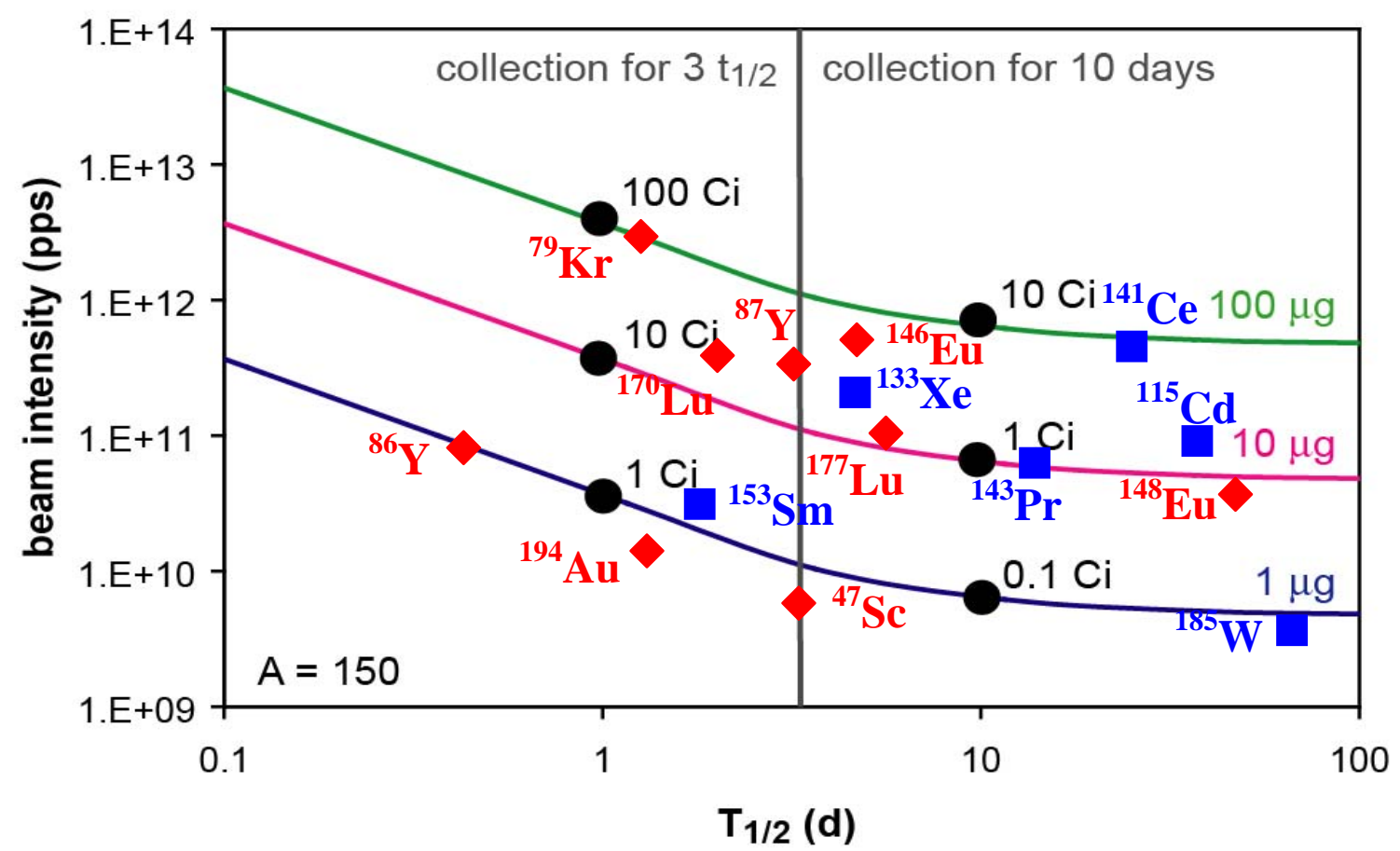

Figure 2: Required production rate versus half-life. The red diamonds are nuclei relevant to stockpile stewardship showing their expected production rates at RIA. The blue squares show the expected production rates for some of the s-process branch points.

Figure 2 shows the relationship between half-life and required production rate for different amounts of required target material. The red diamonds and blue squares show what can be expected at RIA for isotopes relevant to stockpile stewardship and blue diamonds, respectively. The values used were taken from the ANL RIA website [3] for the expected production rates for low energy beams at RIA. As can be seem from the figure, 10 micrograms of many radioactive nuclei can be collected at RIA.

\section{Specifications for Neutron Source Facility}

Generally, this facility must be able to process collected radioactive material into targets for neutron irradiation and provide an intense flux of neutrons from 10's of keV to 20 $\mathrm{MeV}$ in neutron energies. It must also be flexible and have locations for several different experimental setups depending on the reaction of interest and choice in experimental method. It must also be possible to process the sample radiochemically after the neutron irradiation if it is required by the specific experiment. 


\subsection{Radiochemistry Requirements}

Given the production rates of RIA, the upper limit on collected activity of the desired isotope will be roughly $10 \mathrm{Ci}$, which corresponds to collecting the material for one halflife at a rate of $7 \times 10^{12}$ particles/per second. Depending on the production and collection method used, there could be other radioactive contaminants in the collected sample. Thus, the radiochemistry area should be designed to handle $100 \mathrm{Ci}$ of activity. Furthermore, this activity at worst will be hard gamma ray activity. RIA will not be an effective production facility of neutron emitters. Even though the production mechanisms at RIA will provide mass separation, it will still be desirable to have mass separation capability in the radiochemistry area to enhance the isotopic purity and remove contamination produced in the collection process. This is especially true for isotopes collected in the fragmentation line.

\subsection{Energy and Flux Requirements}

This facility must accommodate a variety of neutron reaction cross-section measurements. Different reactions have different neutron energy range of interest thus requiring the facility to provide neutrons over a varied range of energies. The $(n, \gamma)$ crosssection measurements are of most interest for neutron energies between 1-100 keV, while $(n, 2 n)$ cross-sections are of most interest for neutron energies between 10-17 MeV. For some of the charged particle reactions the desired neutron energies extend in the several $\mathrm{MeV}$ range, but there is generally very little interest for neutrons between $100 \mathrm{keV}$ and 3 $\mathrm{MeV}$.

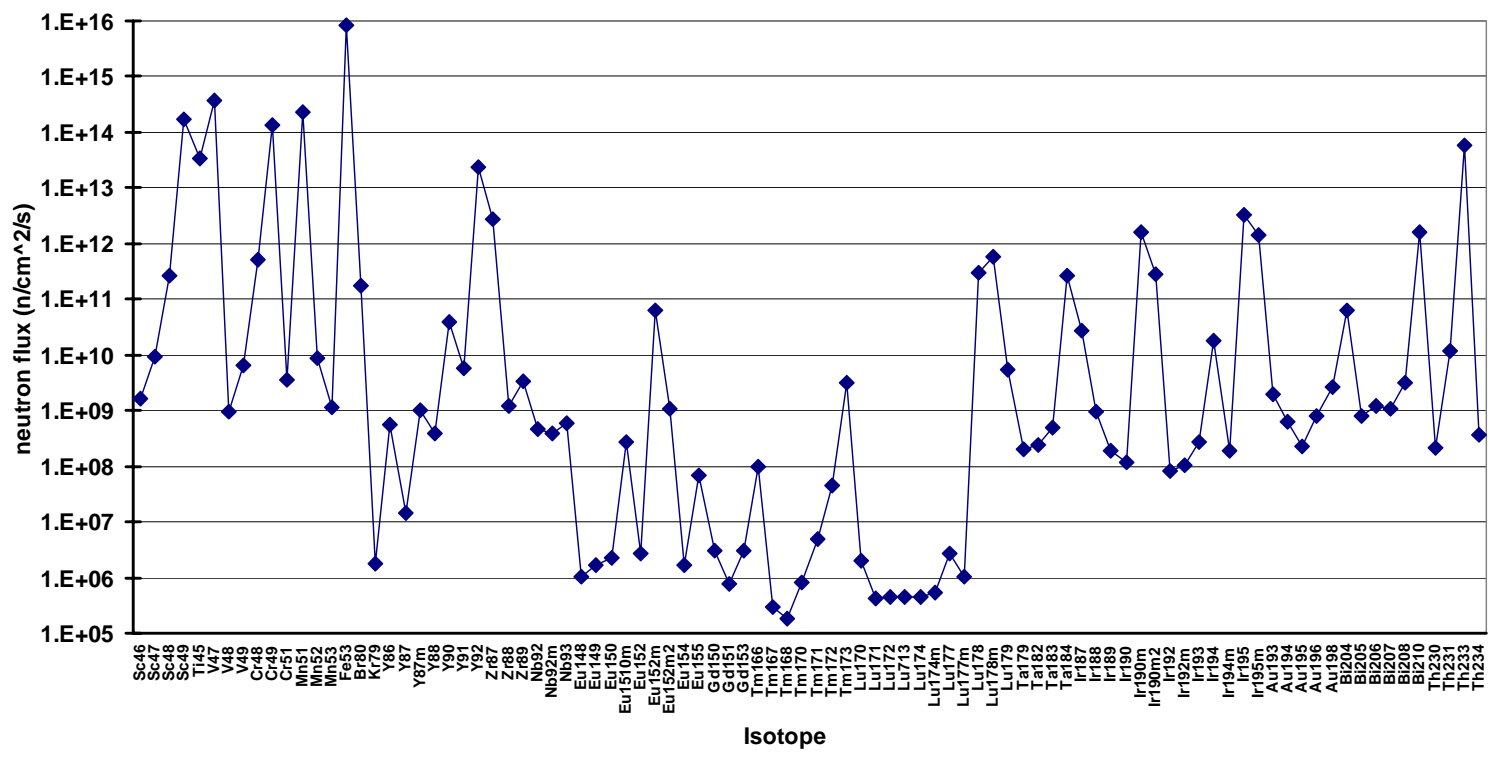

Figure 3: Required Neutron Flux for $(\mathrm{n}, \gamma)$ measurements on unstable nuclei important to Stockpile Stewardship. See text for details.

In general, the higher the neutron flux, the better. Increased neutron flux increases the number of isotopes available for direct measurement and decreases the amount of material required for those that were already possible. Of course the minimum flux 
required will depend on the isotope of interest, the cross-section of the desired reaction, and the choice of measurement technique. Figure 3 illustrates the variance of the required neutron flux for measuring $(n, \gamma)$ cross-sections for many of the unstable nuclei important to stockpile stewardship. It is assumed that the material is collected for 10 days and then irradiated with neutrons for 10 days. The collection rates were taken for reaccelerated beams from the ANL RIA website [3] and the current estimate for the cross-section at $40 \mathrm{keV}$ for each isotope was used. Further, a detection efficiency of $1 \%$ is assumed, typical of activation measurements, and 1000 detected events are required. From this figure, a neutron flux of $10^{10} \mathrm{n} / \mathrm{cm}^{2} / \mathrm{s}$ enables most measurements, but a flux as low at $10^{7} \mathrm{n} / \mathrm{cm}^{2} / \mathrm{s}$ will still be useful. Other reactions and other measurement techniques will have different required neutron fluxes, but the general conclusions taken from this data should still be valid. Figure 4 is the same plot for s-process branch points with the average cross-section for a $30 \mathrm{keV}$ thermal neutron used in the estimate [4].

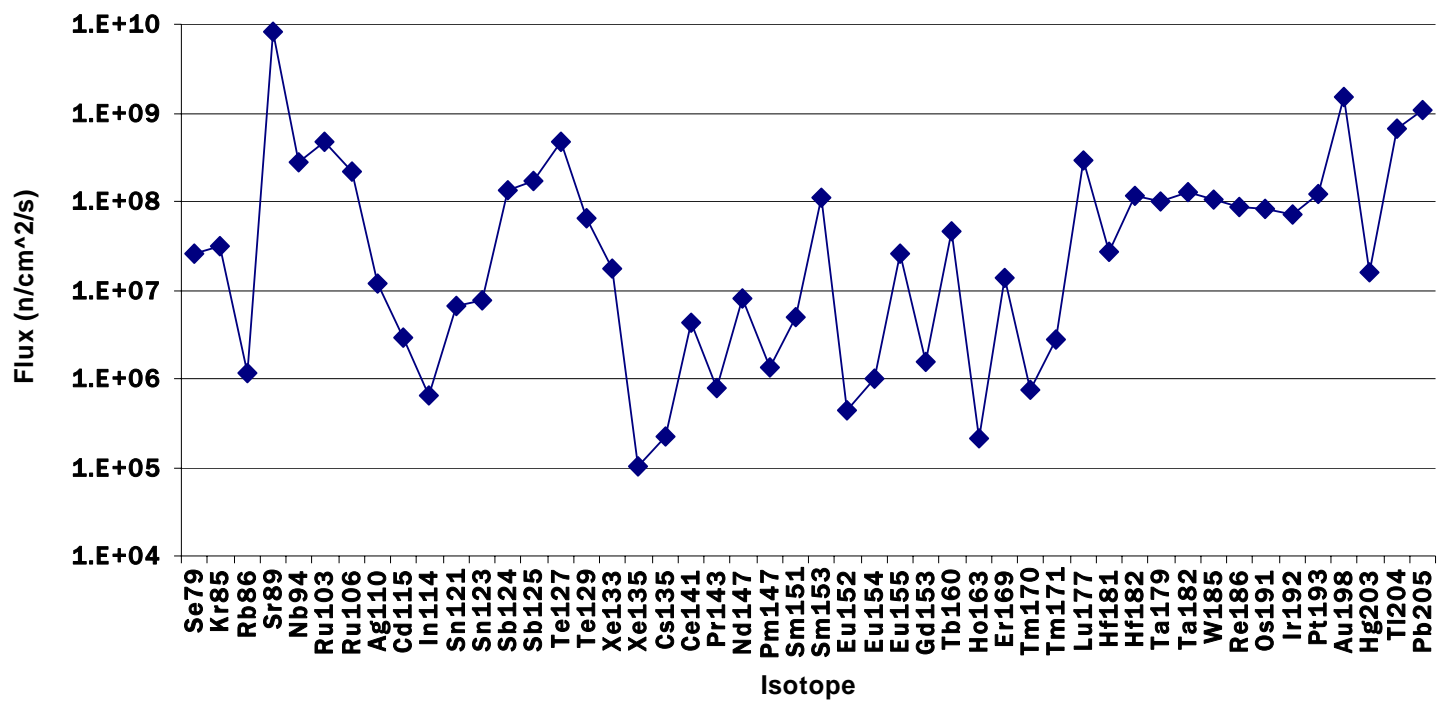

Figure 4: Required Neutron Flux for $(\mathrm{n}, \gamma)$ measurements on s-process branch points. See text for details.

\subsection{Measurement Methods}

In order for the neutron source facility to reach its full utility, it must be able to accommodate several different measurement techniques. Sometimes this implies a dedicated experimental hall for a particular technique or detector setup. Other times the experimental hall must be flexible enough to accommodate several different experimental techniques. The experimental method of choice is not merely a function of neutron beam energy, but also the reaction and species of interest.

\subsubsection{Delayed or Activation Technique}

The simplest measurement method is the activation technique, where a sample is irradiated with neutrons and then the total amount of neutron reaction product is measured. One usually measures a radiation signature of the final product or its daughter, usually gamma rays, though sometimes betas or alphas are the preferred signature. This 
method requires the contamination level of the reaction production prior to neutron irradiation be on the order of $10^{-9}$ or less, because very few target atoms will actually undergo the desired reaction. For $(n, 2 n)$ and $(n, \gamma)$ reactions this issue is compounded because chemical separation is not possible, as the target atoms and reaction product atoms are the same element. For $(n, 2 n)$ measurements, back scattered neutrons are usually not an issue because of the high threshold for those reactions. For $(n, \gamma)$ measurements, back scattered neutrons are a serious concern, often forcing the irradiation to take place in the middle of large rooms, far away from wall, floors and ceilings with as little material as possible around the target. Activation measurements also require at least somewhat "mono-energetic" neutrons.

\subsubsection{Prompt Techniques}

The alternative to activation measurements is prompt measurements in which one tries to measure each reaction as it occurs. The exact technique to use will vary with the reaction

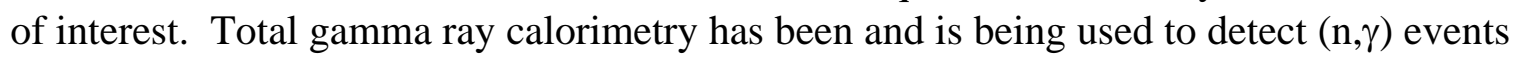
using an array of $\mathrm{BaF}_{2}$ detectors [5-6]. Having a detector at RIA similar to the DANCE array presently at Los Alamos has been studied [7]. The technique requires intense short burst of neutrons with a low duty factor to allow for the background $(n, \gamma)$ event rate to subside before the next neutron pulse. This technique is compatible with using time of flight to determine neutron energy because the low neutron energy implies small path

lengths. Radioactive targets add to the already complicated background issues for this technique.

For (n,xpynza) reactions, some type of spectrometer is envisioned that would detect the charged particle produced in the reaction. Typically these particles would have a few $\mathrm{MeV}$ worth of kinetic energy, and thus would not require intense fields to detect. Bending these charged particles allows one to protect the particle detector from the neutron beam and from the gamma ray background from the radioactive target.

For prompt (n,2n) measurements, the technique is far less developed. The idea would be to detect both emitted neutrons in coincidence. One would need high efficiency neutron detectors and large angular coverage to keep the overall detection efficiency of two neutron events high. The detector system must also be able handle the high gamma ray background coming from the target, which implies a highly segmented array and preferably, neutron detectors that are relatively insensitive to gamma rays. Pulse shape discrimination will also have to be used to help filter out background gamma ray events. Another large background issue is scattered neutrons. The radioactive isotope of interest will be deposited on a backing material, probably a low $\mathrm{Z}$ material with a much higher threshold for $(n, 2 n)$. Neutrons scattering off of this backing material into the detector array must be distinguishable from evaporated neutrons from $(n, 2 n)$ events. Additionally, neutrons scattering off other material in the room and into the detector must be kept to a minimum. This may be facilitated by have an appropriate pulse structure on the beam and applying the corresponding time cut. Frehaut performed many prompt $(n, 2 n)$ measurements on stable targets using a Gadolinium doped scintillator ball which detected the gamma rays as the neutrons capture on Gadolinium [8]. Extending this technique to work with highly radioactive targets is not straightforward, because the large gamma 
background, the small amount of target material, and the much higher required neutron flux present new challenges.

\section{Neutron Source Facility}

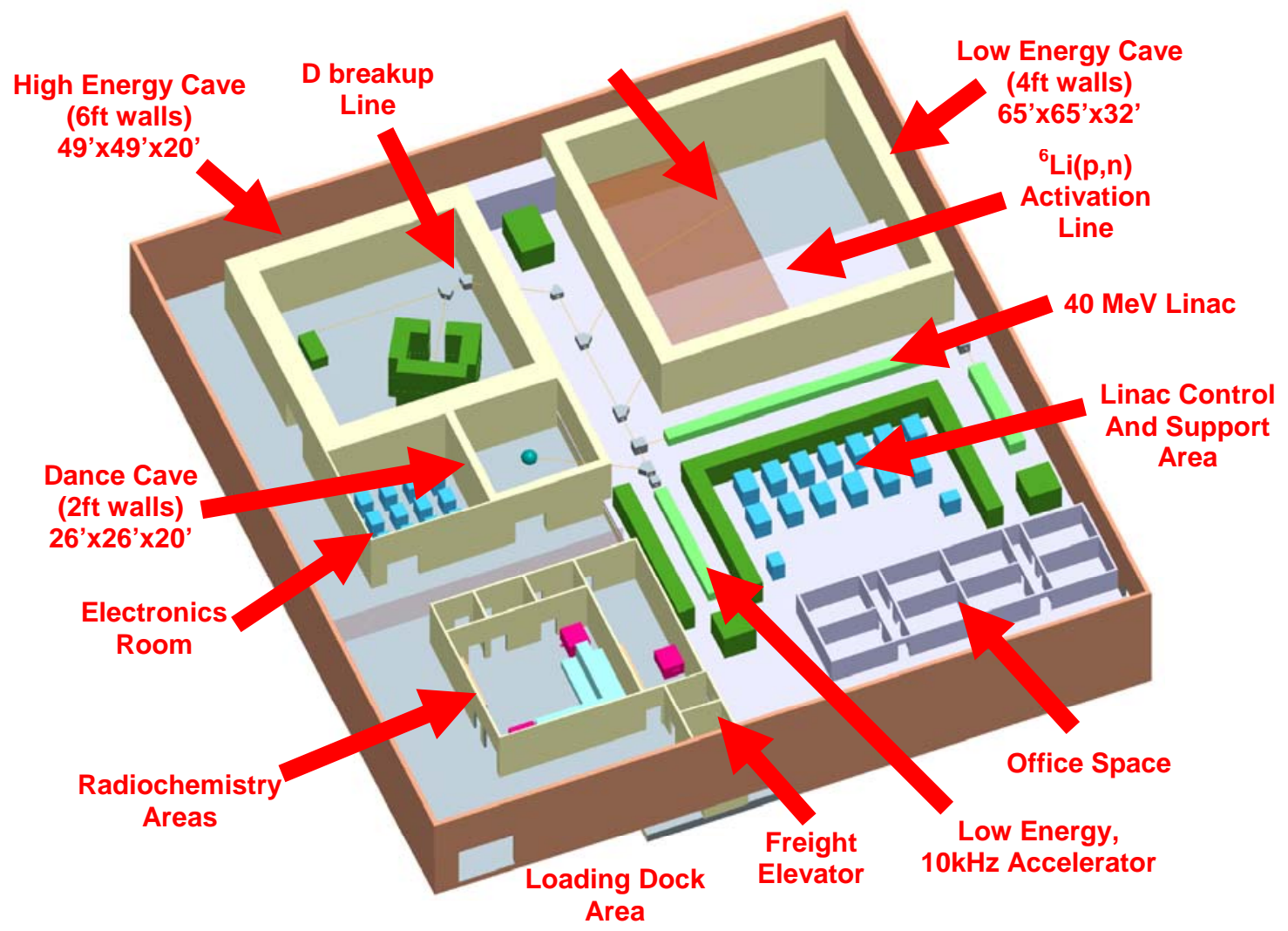

Figure 5: The Neutron Source Facility

In designing a facility to meet the requirements stated in the previous section, one must first determine the method for making the neutrons. Given the neutron energy range and flux required, a spallation neutron source is cost prohibitive. Mono-energetic sources, which use proton or deuteron beams to make neutrons, are the other option. Unfortunately, no single production method covers the entire range, even with flexibility in the choice of energy of the charged-particle beam. Once the production methods are chosen, the number and size of the experimental halls or neutron caves must also be determined. The various experimental techniques, neutron energies, and background considerations all play a role. One must also determine the needed accelerator or accelerators to prepare the charged particle beams of not only the correct current and energy, but timing structure as well. And all of these systems must be integrated with the radiochemistry labs, which process the material received from RIA into targets, and possibly reprocess after neutron irradiation if it is required by the measurement. Considerations such as radiation safety must also be taken into account combining all of these parts into one facility while minimizing space, i.e. costs. Figure 5 shows the current conceptual design of a facility that meets the above requirements. It has two different accelerators, three neutron caves, and radiochemistry facilities, which include a 
mass separator. The details of this design and logic behind the various design choices are discussed below.

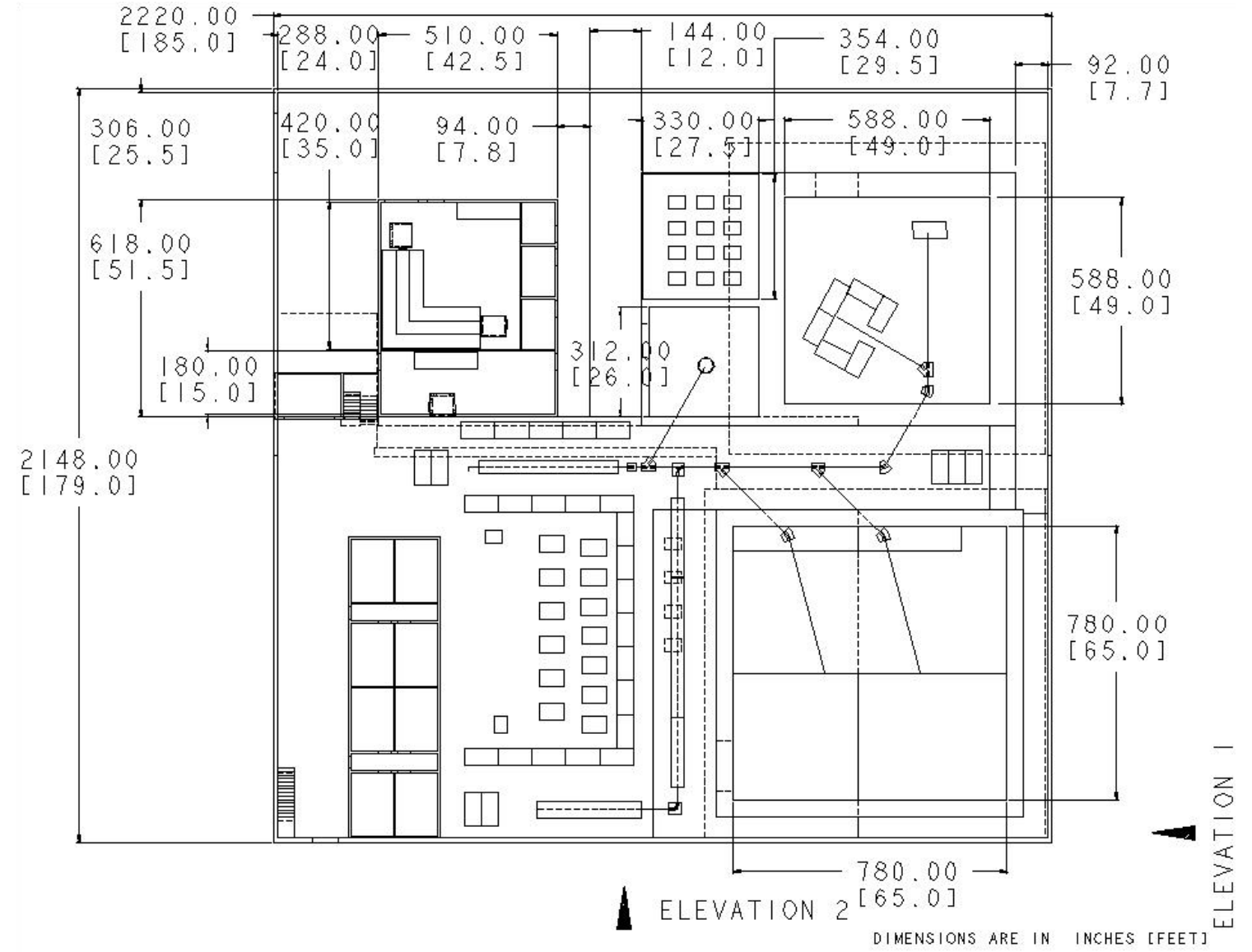

Figure 6: Plan view of Neutron Source Facility.

\subsection{Making Mono-energetic Neutrons}

To make neutrons below several hundred keV in energy, the ${ }^{7} \mathrm{Li}(\mathrm{p}, \mathrm{n})^{7} \mathrm{Be}$ reaction will be used. This reaction has a threshold of $1.88 \mathrm{MeV}$ and a Q-value of $-1.644 \mathrm{MeV}$, making it an excellent choice for neutrons with energies between 5-300 keV. While this reaction could be used to make higher energy neutrons, the cross-section falls off with energy and an excited state in ${ }^{7} \mathrm{Be}$ at $429 \mathrm{keV}$ will cause a bimodal distribution in the neutron energy spectrum. This method does not produce a sharp peak of neutrons as the slowing down of the beam in the target broadens the energy spectrum, but a $1.911 \mathrm{MeV}$ proton beam will produce a neutron spectrum similar to a $30 \mathrm{keV}$ thermal spectrum of neutrons [1], which is relevant to nuclear astrophysics. Also the low neutron energy means short distances are required to use time-of-flight to determine neutron energy. These facts has been exploited at places like Karlsruhe to measure $(n, \gamma)$ cross-sections using both the activation and the total gamma calorimetry technique. Karlsruhe achieved a total neutron yield of $10^{9} \mathrm{n} / \mathrm{s}$ with a $100 \mu \mathrm{A}, 1.911 \mathrm{MeV}$ proton beam [9]. Their target was a thin lithium film deposited on a water cooled copper backing that could withstand up to 150 $\mu \mathrm{A}$. It is hoped by careful design that the beam limit on target can be increased to 1 Amp, but that R\&D challenge is only now starting to be addressed. 
For mono-energetic neutrons from $4 \mathrm{MeV}$ to $12 \mathrm{MeV}$, the $\mathrm{D}(\mathrm{d}, \mathrm{n})^{3} \mathrm{He}$ reaction with a Qvalue of $3.27 \mathrm{MeV}$ can be used quite effectively. One complication for this reaction occurs once the deuteron beam gets above $4.4 \mathrm{MeV}$, when deuteron breakup is possible. The energy of the neutron from break-up, however, is approximately $7 \mathrm{MeV}$ lower than from the $D(d, n)$ reaction. If the reaction of interest has a threshold above the breakup neutron energy, then the breakup neutrons should only cause minor effects in analyzing the data. This type of situation exists for many of the interesting charged particle out reactions. The cross-section for this neutron production reaction is smaller than others (peaking at about 1 mbarns) and requires a gas target, which is inherently thin. It is still hoped that a yield of $10^{10} \mathrm{n} / \mathrm{s}$ per mA of deuteron beam can still be achieved, though like all the production methods mentioned here, there is always a trade-off between neutron energy width and neutron yield.

For neutron energies above $12 \mathrm{MeV}$, the deuteron breakup reaction would be used. The least is know about this production reaction as most of the deuteron breakup data available is for thick targets. Much more should be known about this method over the next year or two as a neutron beam line using this reaction is developed at the 88" cyclotron at LBNL. The target will be solid but whether it should be a low $\mathrm{Z}$ or high $\mathrm{Z}$ target is unclear. High $\mathrm{Z}$ means larger breakup cross-section but also a shorter range for the deuteron beam, smearing out the neutron energy spectrum. It is believed this will be able to produce very intense neutron fluxes, though again it will be a trade-off with the energy spread. If this production method does not prove valid, then one would look into extending the $D(d, n)$ reaction up in energy or use the $T(d, n)^{4}$ He reaction.

\subsection{Neutron Caves}

For neutron production reactions that use beams of less than 8-10 $\mathrm{MeV}$, the primary beam can be stopped in high $\mathrm{Z}$ material directly behind the neutron production target without producing many nuclear reactions or scattering a significant fraction of the neutrons. This is true for the ${ }^{7} \mathrm{Li}(\mathrm{p}, \mathrm{n})$ and $\mathrm{D}(\mathrm{d}, \mathrm{n})$ neutron production reactions. For beams above these energies, some sort of sweeper magnet is needed to separate the primary beam from the secondary neutron beam. The primary beam is then guided to the appropriate beam dump. This is true for the deuteron breakup reaction. To adequately accommodate these two situations, two different experimental areas are required.

The low energy neutron cave would house the beam lines for both ${ }^{7} \mathrm{Li}(\mathrm{p}, \mathrm{n})$ and $\mathrm{D}(\mathrm{d}, \mathrm{n})$ production targets. Activation measurements of these reactions are very sensitive to back scattered neutrons from surrounding material, the walls, floors, and ceilings. The background from the walls decrease by the inverse square of the distance to the walls, thus implying these measurements would need to be in the middle of a large room. As such, we have designed the room to be 65'x 65'x 32', with the beam lines enter the hall halfway between the floor and ceilings. This size in part was selected based on the experience of other facilities [10]. The room will have a false floor to facilitate installation but could be removed if needed to reduce the background during the experiment. These measurements requiring the $\mathrm{D}(\mathrm{d}, \mathrm{n})$ reactions are less sensitive to back scattered neutrons and thus the size of the room is less important. In addition to 
activation measurements, the charged particle spectrometer technique discussed above would also be used on this beam line.

Measurements of $(n, \gamma)$ using total gamma ray calorimetry would not be performed in the low energy cave, but in a separate cave. Given the sensitivity to back scattered neutrons for activation $(n, \gamma)$ measurements, having the array of $\mathrm{BaF}_{2}$ detectors in the low energy hall would not be practical. Also, installation and operations of the array also favor a separate hall for the $\mathrm{BaF}_{2}$ array. As can be seen from figures 5 and 6 , this is the smallest experimental hall. The size of the room is again set to reduce the background, in this case from other neutron capture events in the surrounding material. An electronics room is nearby to house the various electronics necessary for the array and would be accessible during neutron irradiation.

The last neutron cave would be for the high energy neutron experiments that require the deuteron break-up reaction. As stated above, the deuteron beam would not be stopped in the production target and a sweeper magnet would be needed to separate the deuterons and protons from the neutrons. A magnetic field of $1 \mathrm{~T}-\mathrm{m}$ should bend the deuterons by 70 degrees and bend the protons completely around to escape the magnet at 180 degrees. This offers the opportunity the monitor the neutron flux by measuring the produced protons. The beam dump should be reasonably far away from the production target and shielded to avoid neutrons generated at the dump from causing a significant background on the target. Over time, $\mathrm{D}(\mathrm{d}, \mathrm{n})$ reactions will occur at the beam dump, unless the stopped deuterons are allowed to migrate away from the initial stopping depth. Activation, charged particle spectrometer, and neutron coincidence measurements would all be performed in this cave. Whether there would be two separate charged particle spectrometer or just one shared with the low energy cave is yet to be determined. Activation measurements would not be very sensitive to back scattered neutrons since most of the reactions to be measured would have a threshold of several MeV or more.

\begin{tabular}{|c|c|c|}
\hline Cave & Source Estimate & Wall Thickness \\
\hline Low Energy & $10^{11} \mathrm{n} / \mathrm{s}$ & 4 feet \\
\hline Dance & $10^{9} \mathrm{n} / \mathrm{s}$ & 2 feet \\
\hline High Energy & $10^{12} \mathrm{n} / \mathrm{s}$ & 6 feet \\
\hline
\end{tabular}

Table 2: Neutron source used for determining wall thickness, and chosen wall thickness for each neutron cave

In determining the wall thickness for the various caves the following procedure was used. First, an upper estimate was used for the total neutron production rate coming from the various production targets. Table 2 lists what those values are. Second, it was assumed the production target was only 2 meters from the wall and emits neutrons uniformly in space. Generally, 2 meters is much closer than any of the targets are presently envisioned to be, but this allows for safety factors and flexibility in reposition the production targets if need be. Uniform emission is also not generally correct but this usually means correction factors of 2-5, not order of magnitude changes. The wall thickness was then set such that the expected dose rate outside of the wall would be below $50 \mathrm{mR} / \mathrm{hr}$ and less 
than $5 \mathrm{mR} / \mathrm{hr} 5$ feet beyond the wall. This means a fenced off area just outside the cave would be a simple radiological area during operations and the area outside this fence would be a nonradiological area. Table 1 also shows the corresponding wall thickness needed to achieve these goals. Access to cave would be through large doors, which travel on rails and would be large enough for forklifts to bring in heavy equipment.

\subsection{Accelerator}

The broad range of neutron requirements needed to meet the experimental physics objectives of the neutron source facility put significant demands on the accelerator technology needed to drive the production targets. The first requirement comes in wanting to produce monoenergetic neutrons covering an energy range of 0.005 to 20 $\mathrm{MeV}$. While producing neutrons covering a broad energy range is commonly done in spallation neutron sources [11], to extract and use neutrons of a certain energy with a narrow energy bandwidth makes this approach undesirable because of the large distances needed to discriminate the desired-energy neutrons from the rest by time-of-flight spectroscopy. This approach is also inherently less efficient since high energy driver accelerators are needed, and much of the beam power goes into generating neutrons at all energies, most of which are not of interest.

The second requirement comes from the need of high neutron intensities. Since higher source intensity allows shorter half-life species to be measured, making this facility most cost effective in obtaining neutron cross-section data for species far from stability means pushing the neutron sources to the highest intensities possible. The third requirement comes from the nature of monoenergetic neutron production targets. Monoenergetic neutrons are a product of a nuclear interaction between an incoming ion $\left(\mathrm{p}^{+}\right.$or $\left.\mathrm{d}^{+}\right)$and a target nucleus (e.g., deuterium, tritium, or lithium-7), and are typically monoenergetic only over limited to narrow energy ranges. This is due to either the presence of low-lying excited states that give rise to bimodal energy distributions, or due to the variation in cross sections with energy for competing reactions coming up and broadening or swamping the original monoenergetic output.

The fourth requirement comes from the need for different pulsed neutron timing for different measurement techniques. For most higher energy measurements ( $>4 \mathrm{MeV})$, it is most important for the time-integrated neutron intensity coming out of the target and going through the sample to the detector be as high as possible. This translates to wanting accelerators that can deliver high average currents beams. Other, lower energy measurements, i.e., DANCE, need very short ( 1 nsec), very high intensity ( 0.1-5 A peak current) beam pulses spaced by $\sim 100$ usec to allow the detectors to clear. This translates to needing very high peak current beams at very high rep rates and very low duty factors.

The final requirement comes from needing near-continuous energy variability out of the neutron source driver accelerators. To achieve monoenergetic neutrons in a nearcontinuous range out of the neutron sources requires the drivers to have an energy agility that is very atypical for an resonant-RF accelerator. This presents an appreciable 
technical challenge, especially when it is combined with the other requirements. A summation of the neutron production and accelerator requirements is shown in Figure 7.

\section{RIA NEUTRON SOURCE - Neutron Production}

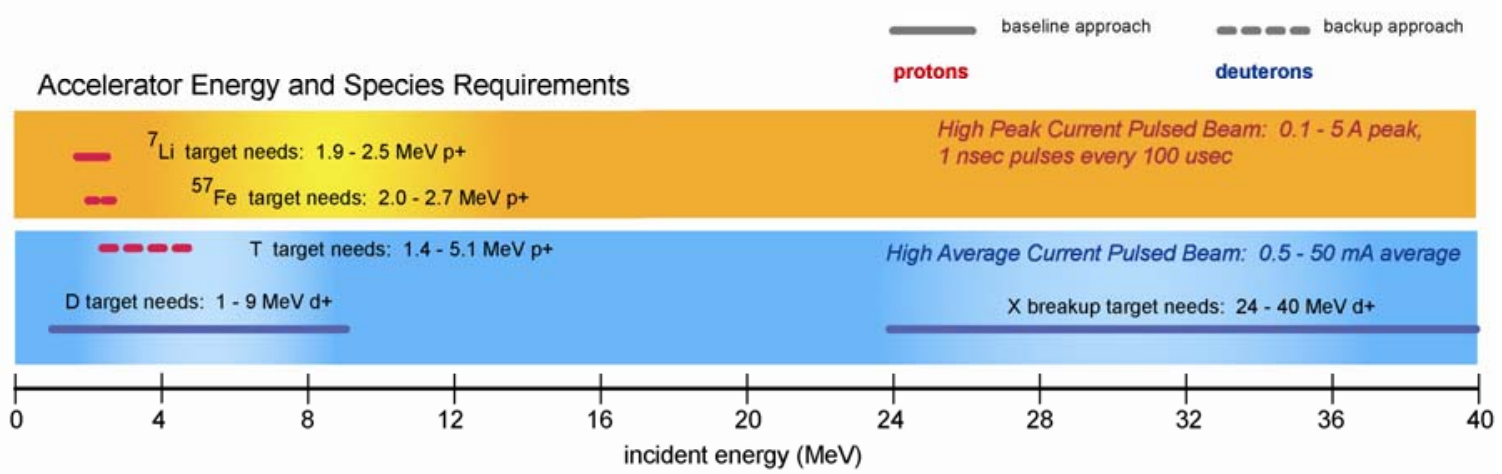

Monoenergetic Neutrons Produced

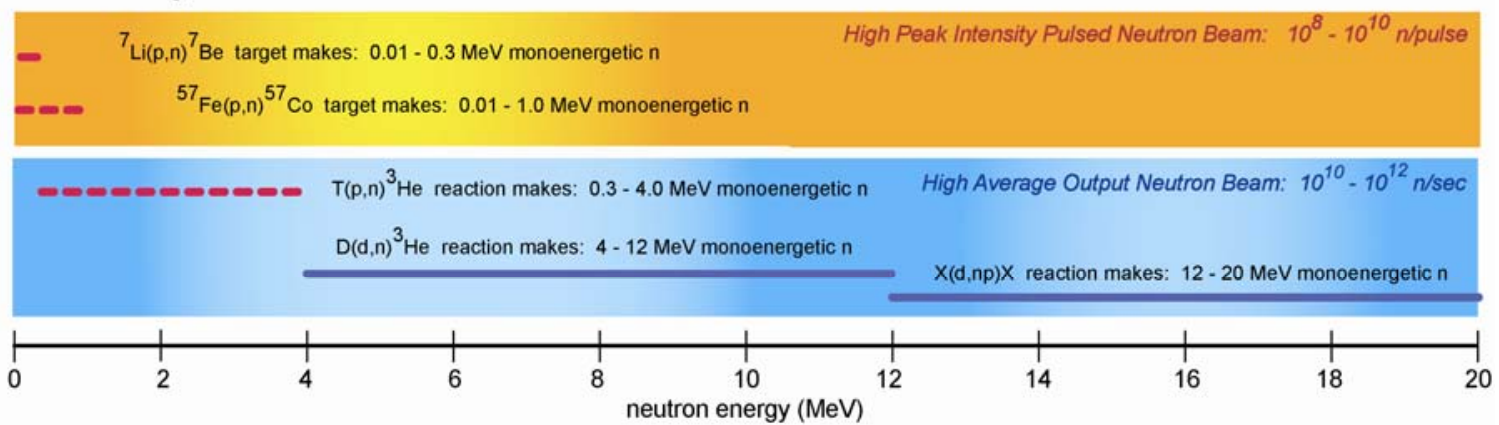

Figure 7: Graphic showing the accelerator constraints (top) and neutron production requirements (bottom).

A technology evaluation was done to determine candidate design approaches that could best meet the constraints outlined above. A survey was done that spanned the extent of accelerator technology. The results are shown in Table 3.

Out of this evaluation came a number of "strawman" designs that were roughly costed and scoped for comparison purposes. Both normal conducting and superconducting RF accelerator types were evaluated as part of the study. The conclusion at this stage is that a combination of two accelerators will be needed to meet the requirements as they are presently envisioned - one that is energy-agile and capable of accelerating high average currents to $40 \mathrm{MeV}$ and can accelerate either protons or deuterons with minimal retuning impact, and a second, low energy, high peak current proton machine that can deliver beam in the timing structure desired.

For the high average current machine, the leading candidate design is to take the basic front end design of the RIA driver linac and rework it into a "high-output" (HO) configuration. This would mean increasing the power coupler and RF amplifier capacity to accelerate up to $25 \mathrm{~mA}$ of beam $\mathrm{CW}$, as well as confirming the focusing lattice could handle the increased space charge forces. This approach benefits from all the design and 
beam dynamics studies done thus far on the RIA driver linac, as the cavities and lattice can pretty much be build to print from the drawings being done for RIA. The impact of the higher power coupler would need to be evaluated further. This machine would suit the neutron source facility very well as it is an inherently energy-agile machine (it was designed that way from the start), and runs at a low frequency (bunch repetition is at 57.5 $\mathrm{MHz}$ ) which gives more charges per bunch and better accommodates chopping.

\begin{tabular}{|l|l|l|l|}
\hline & \multicolumn{1}{|c|}{ Pros } & \multicolumn{1}{c|}{ cons } & \multicolumn{1}{c|}{ comment } \\
\hline HIGH AVERAGE CURRENT APPROACHES & $\begin{array}{l}\text { can use RIA driver } \\
\text { design }\end{array}$ \\
\hline $\begin{array}{l}\text { SCRF heavy-ion } \\
\text { linac at 57-175 MHz }\end{array}$ & $\begin{array}{l}\text { reliable, proven, } \\
\text { energy variable, } \\
\text { efficient }\end{array}$ & cryogenic, complex \\
\hline $\begin{array}{l}\text { NC-RF linac at } \\
\text { 425 MHz }\end{array}$ & reliable, proven & $\begin{array}{l}\text { need high beam } \\
\text { loading for efficiency }\end{array}$ & $\begin{array}{l}\text { less energy agile, } \\
\text { new development }\end{array}$ \\
\hline Pelletron & $\begin{array}{l}\text { good energy agility, } \\
\text { DC beam }\end{array}$ & low current, size & $\begin{array}{l}\text { DC beam precludes } \\
\text { windowless aperture }\end{array}$ \\
\hline Cyclotron at 25 MHz & $\begin{array}{l}\text { provides 1 nsec } \\
\text { pulses at 100 nsec }\end{array}$ & $\begin{array}{l}\text { low current, limited } \\
\text { energy agility, big }\end{array}$ & $\begin{array}{l}\text { low frequency good, } \\
\text { but not for variability }\end{array}$ \\
\hline HIGH PEAK CURRENT APPROACHES & \multicolumn{2}{|l|}{} \\
\hline Induction linac & $\begin{array}{l}\text { potential for very } \\
\text { high peak current }\end{array}$ & $\begin{array}{l}\text { Limited experience } \\
\text { with ions }\end{array}$ & $\begin{array}{l}\text { worth studying } \\
\text { further }\end{array}$ \\
\hline $\begin{array}{l}\text { Rapid cycling } \\
\text { synchrotron (RCS) }\end{array}$ & $\begin{array}{l}\text { could provide ideal } \\
\text { beam pulse structure }\end{array}$ & $\begin{array}{l}\text { underdeveloped, } \\
\text { large size }\end{array}$ & $\begin{array}{l}\text { may be worthy } \\
\text { backup, needs study }\end{array}$ \\
\hline $\begin{array}{l}\text { Dynamitron } \\
\text { good energy agility, } \\
\text { AC CW beam }\end{array}$ & $\begin{array}{l}\text { low current, } \\
\text { unproven for ions }\end{array}$ & $\begin{array}{l}\text { AC-CW beam still } \\
\text { needs to be chopped }\end{array}$ \\
\hline
\end{tabular}

Table 3: A summary of the accelerator technologies evaluated for study.

The high peak current machine is a significantly greater technical challenge. Presently, there is no obvious machine approach that can match the requirements of a 0.1-5 mA peak current beam at a $10 \mathrm{kHz}$ rep rate. While the timing structure can be achieved in a number of different accelerator types, most all of these require chopping a beam, which severely impacts efficiency and overall beam intensity delivered. Two possibilities exist for achieving such a timing structure: proton storage rings and induction linacs.

A compact proton storage ring could be used to store and build up charge that is then dumped to the DANCE detector at prescribed intervals. This approach would involve accelerating an $\mathrm{H}$ - beam that would then be injected and stored in a ring. The size and complexity of this approach need further evaluation to demonstrate its efficacy in this application.

Induction linac technology appears to offer the possibility of generating such a beam as well, and it has a proven track record of accelerating kiloampere bunches of electron beams [12], and is being studies extensively for potential heavy ion fusion applications [13]. But, to date, there is limited experience with intense proton beams accelerated in 
this type of machine. To better understand this application space, further evaluation and study is planned for FY05.

\subsection{Radiochemistry Facilities}

In order to receive and handle the 10 s of micrograms of short-lived, radioactive isotopes made by RIA, the neutron source will need a radiochemistry facility in addition to the accelerators and neutron target areas. The radiochemistry area will house equipment capable of allowing the physical manipulation of radioactive materials that will have hard gamma radiation levels of $\sim 10 \mathrm{Ci}$ or more.

The current plan is to build three hot cells all with remote manipulators for radiochemists to use for manipulating the material. The general design for each hot cell will be that of a shielded, open-faced, 5-sided box with an optically-accurate shielding window that can swing down below the box workspace for pre-handling preparation and post-handling clean up. Each box will be roughly 5 feet wide, 3 to 4 feet tall, and roughly 4 feet deep. They will be 'non-closed' (non-pressured) systems, each with its own negative-flow, filtered-exhaust ventilation system. Each box should have at least 5 feet of open space around it and its shielding for any potential chemical processing equipment that can be located outside of the box, i.e., inert gas bottles, acid/base reservoirs, other reagents, etc. that can be 'piped' into the box from the outside. This allows more space inside and keeps these materials from being contaminated with radioactive materials. Most, if not all, physical and chemical manipulation of the radioactive sample will be done using remote manipulators that are accessible to the radiochemist at the front window but will penetrate the box from the topside down into the hot cell. The inside of the box should be capable of being equipped with an assortment of chemistry equipment and mechanical apparatus such that 3 fundamental, physical processes can occur: 1. dismantling of the RIA target/holder assembly, 2. processing of the target for the isotope of interest, and 3. preparation of a secondary target for neutron bombardment.

Dismantling the RIA target/holder will include physical manipulation using tools such as wrenches, screwdrivers, tweezers, etc. Both processing and preparation of a secondary target will include the chemical and possibly the physical manipulation of the material. Chemical processes that are anticipated include but should not be limited to crushing, dissolving, electroplating, column separations, liquid-liquid separations, precipitation, collection (sedimentation or centrifugation), pressing, etc. Each isotope of interest will likely require a unique series of steps, a specific recipe or procedure that is, to get from process 1 through process 3; however, many of the individual steps will be of a generic form that can be used in any order necessary as the case requires.

One significant piece of equipment to be housed in the radiochemistry facility will be an isotope separator. It will be housed in a room that has 2 hot cells. This room will serve as receival area of RIA materials and processing steps 1, 2, and 3 described in the preceding paragraph. Both hot cells will be similarly equipped, but one will be positioned near the input end of the isotope separator and the other at the output end of the isotope separator. How the separator and hot cell are connected has yet to be fully defined, but it is anticipated that either the hot cells can be elongated on one side so as to 
include the input/output hardware of the separator or to add some type of antechamber to a hot cell that allows for periodic connectivity with the input/output hardware while retaining a way to physically segregate the hot cell from the input/output hardware when not in use. If isotope separation is not required for a measurement, then either hot cell can be used for secondary target preparation. This somewhat large room will also have bench tops and fume hoods for preparatory chemistry that would have to be done prior to doing the actual sample chemistry in the hot cells. Adjacent to this room, will be two smaller room: one to house the power supply and associated equipment related to the isotope separator and the other a control room for operating the isotope separator.

In addition to these rooms, the RadChem facility will have one more hot cell room that contains 1 additional hot cell that will be completely separate from the 'receival' room. This lab will be for any potential, post-neutron-bombardment chemistry or physical manipulation that may need to be performed on the secondary target material prior to measurement of the reaction products. Currently, one hot cell is planned, but a second, redundant cell would be useful in the event of equipment failure or inadvertent contamination of the cell that may threaten the use of the cell in a timely fashion for the next planned experiment. This room will also have bench tops and fume hoods for any preparatory chemistry that would need to be performed.

Finally, a fifth room would be included in the RadChem facility. This would simply be a small, shielded room for the storage of radioactive materials. These would include wastes that need to cool prior to disposal and post-neutron-bombarded samples that need to have parent products decay away before the desired material can be quantified.

Radioactive sample transport to and within the facility is another issue to be addressed. Currently, the plan is to implement a forced-air type rabbit system to push targets from station to station. This would possibly include have samples sent from RIA to a 'receival' hot cell in RadChem section of the Neutron Source Facility. From there, the processed samples would be shuttled to the various neutron target areas through a similar system. And, if post neutron irradiation chemistry is to be performed, the transport system will have to be able to return the material to the second hot cell area in the RadChem facility. Also, the facility will be able to receive material from RIA that has been transported by truck in a shielded, 'pig'-type of canister. There will be a loading dock doors at the neutron facility large enough to access a trucked in shipment from inside with a forklift together with interior alleyways and doors large enough to maneuver the pig into the receival room in the RadChem facility. One or both hot cells will have another small antechamber specifically designed to receive these types of shielded packages and remove and transport the radioactive material into the hot cell while shielding radiochemists during the process. From there, the in house rabbit system will again transport samples from place to place.

\subsection{Budget}

Table 4 below shows the estimated budget for this facility. The costs are broken into building and conventional facilities, accelerators, radiochemistry areas, and experimental areas. An overall contingency was added to the estimated. A 30\% contingency was 
added for the facility costs and a 50\% contingency to the experimental areas because of the larger uncertainty for the detector systems that will go in the neutron caves. For the concrete, a cost of $\$ 400$ dollars per cubic yard installed was used for the wall and ceilings of the concrete caves, while a cost of $\$ 200$ dollars per cubic yard installed was used for the floors. The loading on the floors should be less than the soil load limit such that the excavation and foundation pour should be straightforward. For the RF accelerator, it is assumed that the cavities will be the same as the main RIA linac so that limited R\&D will be needed. Also, it is assumed that this facility will be able to tie into the main cryogenics plant at RIA. The costs for the radiochemistry hardware are based on similar equipment that has been used at LLNL in the past and design studies of installing a mass separator at LLNL. The costs for experimental equipment are based on past experience with similar detector systems.

\begin{tabular}{|c|c|c|c|c|c|}
\hline 1 & Building and Conventional Faciliites & & & & 17043 \\
\hline 1.1 & Earthwork & & & 1000 & \\
\hline 1.2 & Concrete & & & 9010 & \\
\hline 1.2.1 & Floors & & 3030 & & \\
\hline 1.2.1.1 & Low Energy Cave & 690 & & & \\
\hline 1.2.1.2 & High Energy Cave & 740 & & & \\
\hline 1.2.1.3 & Rest of floor & 1600 & & & \\
\hline 1.2.2 & Cave Walls and Ceilings & & 5980 & & \\
\hline 1.2.2.1 & Low Energy Cave & 2500 & & & \\
\hline 1.2.2.2 & High Energy Cave & 2200 & & & \\
\hline 1.2.2.3 & Dance Cave & 280 & & & \\
\hline 1.2.2.4 & Accelerator & 1000 & & & \\
\hline 1.3 & Outer Building & & & 1000 & \\
\hline 1.3.1 & Walls & & 500 & & \\
\hline 1.3.2 & Roof & & 500 & & \\
\hline 1.4 & Interior Rooms & & & 900 & \\
\hline 1.4.1 & Accelerator Control Room & & 100 & & \\
\hline 1.4.2 & Office Space & & 100 & & \\
\hline 1.4 .3 & Radiochemistry Area & & 500 & & \\
\hline 1.4.4 & Waste Storage Area & & 200 & & \\
\hline 1.5 & Utilities & & & 600 & \\
\hline 1.5.1 & Electrical & & 200 & & \\
\hline 1.5 .2 & Plumbing & & 200 & & \\
\hline 1.5 .3 & Venting Systems & & 200 & & \\
\hline 1.6 & Safety Systems & & & 600 & \\
\hline 1.6.1 & Fire & & 200 & & \\
\hline 1.6 .2 & Radiation & & 400 & & \\
\hline 1.7 & Contingency (30\%) & & & 3933 & \\
\hline 2 & Accelerators & & & & 36226 \\
\hline 2.1 & Low Energy Accelerator & & & 5000 & \\
\hline 2.2 & High Energy Accelerator & & & 22866 & \\
\hline 2.2.1 & Ion Source & & 120 & & \\
\hline 2.2.2 & LEBT & & 195 & & \\
\hline 2.2 .3 & LINAC & & 7751 & & \\
\hline
\end{tabular}


$\begin{array}{llrr}\text { 2.2.3.1 } & \text { Accelerating Structure } & 6410 & \\ \text { 2.2.3.2 } & \text { RF Generators } & 1341 & \\ \text { 2.2.4 } & \text { Cryostat } & & 3800 \\ \text { 2.2.4.1 } & \text { Structure } & 2750 & \\ \text { 2.2.4.2 } & \text { Magnet } & 600 & \\ \text { 2.2.4.3 } & \text { Transfer Lines } & 450 & \\ \text { 2.2.5 } & \text { HEBT } & & 3500 \\ 2.2 .6 & \text { Installation } & & 3000 \\ \text { 2.2.7 } & \text { Design Engineering } & & 4500\end{array}$

2.3 Contingency (30\%) 8360

3 Radiochemistry Area

3.1 Hot Cells

3.2 Transfer Area

3.3 Mass Separator

3.4 general lab facilities

3.5 Ventilation

3.6 Transport System within Facility 5000

$\begin{array}{lll}3.7 & \text { Contingency (30\%) } & 5670\end{array}$

4 Experimental Areas

4.1 DANCE Cave

$\begin{array}{rrr} & & 24570 \\ & 2000 & \\ & 500 & \\ & 10000 & \\ & 400 & \\ & 1000 & \\ & 5000 & \\ & 5670 & \\ & & \\ & & 22650 \\ & 5800 & \\ 300 & & \\ 5000 & & \\ 500 & & \\ & 2000 & \\ 200 & & \\ 300 & & \\ 700 & & \\ 300 & & \\ 500 & & \\ 300 & 7300 \\ 500 & \\ 1000 & \\ 5000 & \\ 500 & & \\ & & \\ & & \end{array}$

4.1.1 Lithium Target 300

4.1.2 Detector 5000

4.1.3 Other Equipment 500

$\begin{array}{lll}4.2 & \text { Low Energy Cave } & \\ 4.2 .1 & \text { Beam Magnet } & 200\end{array}$

4.2.2 Lithium Target 300

4.2.3 Deuteron Target 700

4.2.4 Charged Particle Spectrometer 300

4.2.5 Other Equipment 500

4.3 High Energy Cave 7300

4.3.1 Beam Magnet 300

4.3.2 Separator Magnet 500

4.3.3 Deuteron Beam Dump 1000

4.3.4 Detector System $\quad 5000$

4.3.5 Other Equipment 500

4.4 Contingency (50\%) 7550

Table 4: Preliminary Costs Estimate. Values are $\mathrm{k} \$$.

\section{Examples}

This section contains several examples of how direct neutron cross-section measurements on radioactive targets can be done at RIA if the proposed facility presented in this document is on site. The examples cover astrophysics and stockpile stewardship measurements. The examples also try to cover all the experimental techniques, illustrating the issues for each technique and possible solutions. This is by no means a 
comprehensive list of all situations that could potentially arise in trying to do these measurements, as each case must be studied in detail. However, it does illustrate how some of the vast scientific potential can be achieved at RIA.

\subsection{Astrophysics $-{ }^{60} \mathrm{Fe}(n, \gamma)$}

An example of an astrophysical interesting isotope that could be measured via the activation technique, provided the sample would be available, is ${ }^{60} \mathrm{Fe}$. The half-life of

${ }^{60} \mathrm{Fe}$ is $7.510^{6}$ years and has an expected production rate at RIA between $10^{8}$ and $10^{10}$ particles per second. It should only take a few days to collect enough material for a target and there is ample time for processing and neutron irradiation. Equally important, the decay of ${ }^{60} \mathrm{Fe}$ produces only low energy betas (end point energy of $180 \mathrm{keV}$ ) and a single gamma ray with energy of $60 \mathrm{keV}$. Additionally, this gamma ray is only emitted for $2 \%$ of the decays. However, when the neutron capture product, ${ }^{61} \mathrm{Fe}$ with a half-life of $6 \mathrm{~min}$, decays, it releases high energy gamma rays, which can be detected applying the so-called cyclic activation technique. It has been shown in the past that such measurements are possible with as few as $10^{14}$ atoms [14]. Therefore, a neutron capture cross section measurement on ${ }^{60} \mathrm{Fe}$ should therefore be possible at RIA.

\subsection{Astrophysics - ${ }^{141} \mathrm{Ce}(n, \gamma)$}

As mentioned above, the neutron capture cross-section for most branch point nuclei cannot be measured via the activation technique because the capture product has no radiation signature for detection. ${ }^{141} \mathrm{Ce}$ is one such nuclei, as ${ }^{142} \mathrm{Ce}$ is stable. For nuclei such as these, using total gamma ray calorimetry with a detector like DANCE is the only option for direct measurements. In this case, the production rate at RIA is quite high, $5 \times 10^{11}$ particles per second, allowing for a target of $3.9 \times 10^{17}$ atoms after 10 days of collection. Given a conservative neutron flux of $10^{7} \mathrm{n} / \mathrm{cm}^{2} / \mathrm{sec}$, an expected cross-section at $40 \mathrm{keV}$ of $120 \mathrm{mb}$, and a 10 day irradiation time, then $3 \times 10^{5}$ neutron capture events would occur during the experiment. A DANCE like array has a nearly $100 \%$ efficiency for detecting neutron capture events, but is often difficult to measure the exact number of neutron capture events due to background from other neutron capture event and the decaying target. Given a half-life of 32.5 days, then the target will have an activity of 2.6 Ci. When ${ }^{141}$ Ce decays, the only gamma radiation that is released is a $145.4 \mathrm{keV}$ gamma ray with a branching ratio of only $48.2 \%$. This results in $4.6 \times 10^{10}$ gamma rays per second will be released from the target. Fortunately, the gamma rays that are emitted from a neutron capture event are typically of a much higher in energy, several $\mathrm{MeV}$, allowing the opportunity to use shielding for target background radiation. In earlier simulations, the team involved with DANCE has looked at using $5 \mathrm{~mm}$ of $\mathrm{Pb}$ to shield against low energy gammas from the target [2]. While this only reduces the neutron capture efficiency by less than $2 \%$, the background gammas from ${ }^{141} \mathrm{Ce}$ are attenuated by $2 \times 10^{-7}$. Thus, in this configuration only $9.2 \times 10^{3}$ gamma rays per second would be incident on the detector array of some 150 or more modules, implying each detector would see a background rate of 60 gamma rays per second. The digitizing time scales involved are $1 \mathrm{~ns}$ for the fast decay component and $2 \mu$ s for the slow decay component, making target decay background not an issue. In fact one could reduce the shield by a factor of 2 and still perform the measurement. Given the expected number of good 
events, one could also reduce the amount of target material by a factor of 10 and possibly more, depending on the desired accuracy.

\subsection{Stockpile Stewardship - ${ }^{86} Y(n, 2 n)$}

Given the expected production rates at RIA and if a flux for $14 \mathrm{MeV}$ neutrons of $5 \times 10^{9}$ $\mathrm{n} / \mathrm{cm}^{2} / \mathrm{s}$ can be achieved, then it should be possible measure the ${ }^{86} \mathrm{Y}(\mathrm{n}, 2 \mathrm{n})$ cross-section via the activation technique. Given the half-life of ${ }^{86} \mathrm{Y}, 14.74$ hours, a target of only $5.4 \times 10^{15}$ atoms can be made at RIA. The reaction product, ${ }^{85} \mathrm{Y}$, is even shorter lived, but its daughter, ${ }^{85} \mathrm{Sr}$, has a 64.8 day half-life, which allows the use of chemistry and time to increase the signal to noise. Thus, the procedure of the experiment would be the following: 1) irradiate the target for a length of time that maximizes the amount of ${ }^{85} \mathrm{Y}$ in the target, 2) chemically separate Sr from the target and remove, 3) wait 20 days for the ${ }^{86} \mathrm{Y}$ to decay to stable ${ }^{86} \mathrm{Sr}$, and 4) Measure the $514 \mathrm{keV}$ gamma of ${ }^{85} \mathrm{Sr}$ decay. The ground state of ${ }^{85} \mathrm{Y}$ has a half-life of 2.6 hours while the first excited state has a half-life of 4.9 hours with both states decaying into ${ }^{85} \mathrm{Sr}$ nearly $100 \%$ of the time. The quantity of these two states is maximized after about 10 hours of neutron irradiation, with about $7.4 \times 10^{4}{ }^{85} \mathrm{Y}$ atoms in the ground state and $1.2 \times 10^{5}$ atoms in the excited state. Given the various branching ratios, about $90 \%$ of theses atoms will result in a release of a $514 \mathrm{keV}$ gamma ray from a ${ }^{85} \mathrm{Sr}$ decay. Immediately after neutron irradiation, the target will already contain a sample ${ }^{85} \mathrm{Sr}$ from previously decays ${ }^{85} \mathrm{Y}$ and from the ${ }^{86} \mathrm{Y}(\mathrm{n}, \mathrm{np})$ reaction. Thus, an immediate chemical separation must be done, after which all subsequent ${ }^{85} \mathrm{Sr}$ in the target will be from ${ }^{86} \mathrm{Y}(\mathrm{n}, 2 \mathrm{n})$ reactions alone. Waiting 20 days after the separation is necessary to allow for the ${ }^{86} \mathrm{Y}$ to decay away and remove its initially overwhelming background. It will necessary to count specifically for its decays during those 20 days to know how much target material was present. But after the 20 days, which causes a negligible loss of signal, one can then count the sample for ${ }^{85} \mathrm{Sr}$ decay. Assuming an overall efficiency of $2 \%$ then, 4000 events should be detected over the course of a 64 day count. This count rate should be well above room background and allow for the measurement of the ${ }^{86} \mathrm{Y}(\mathrm{n}, 2 \mathrm{n})$ cross-section.

\subsection{Stockpile Stewardship - ${ }^{48} V(n, \alpha)$}

The half-life of ${ }^{48} \mathrm{~V}$ is 15.9 days but ${ }^{45} \mathrm{Sc}$, the reaction production of the $(n, \alpha)$ reaction, is stable, thus making the activation method impractical for this measurement. It should however, be possible to detect the alpha particle from the reaction. The coulomb barrier between ${ }^{45} \mathrm{Sc}$ and an alpha particle is about $8 \mathrm{MeV}$, while the $\mathrm{Q}$ value of the reaction is 2.2 MeV. This implies the cross-section is not significant for neutron energies below 6 $\mathrm{MeV}$ and the alpha particles released from the reaction will have at least $8 \mathrm{MeV}$. Detecting the alpha particle is relatively straight forward with any number of particle detectors, such as semiconductors. Identifying from other charged particles is harder but can be done by using magnetic and/or electric fields as a charge to mass analyzer. Time of flight could also be used to help determine particle identification as an $8 \mathrm{MeV}$ alpha as a velocity of $2 \mathrm{~cm} / \mathrm{ns}$. Using time of flight however would require a pulsing the accelerator resulting in a reduction of neutron intensity. 


\section{Summary}

RIA presents the best and only opportunity to make many neutron cross section measurements important to stockpile stewardship and astrophysics. These measurements involve collecting radioactive nuclei at RIA, processing the sample into a target with radiochemistry capability, and putting the target in front on an intense, "mono-energetic", energy tunable neutron flux. A preliminary conceptual design of the required neutron source with radiochemistry capabilities has been preformed. The current design has three experimental areas and two different accelerators in order to accommodate the variety of neutron energies and measurement techniques. While this facility will be on the same site, its impact on design and operations of RIA should be minimal.

\section{References}

1. C. L. Jiang, et al, NIMA, 492, (2002) 57-73.

2. http://www.sc.doe.gov/henp/np/nsac/docs/RIA-GSI-nsac-022604.pdf.

3. http://www.phy.anl.gov/ria/w.html.

4. $\quad$ Z.Y. Bao, et al, Atomic Data and Nuclear Data Tables, 76, (2000) 70-154.

5. $\quad$ K. Wisshak, et al, NIMA, 292, (1990) 595-618.

6. M. Heil, et al, NIMA, 459, (2001) 229-246.

7. R. Reifarth, et al., NIMA, 524, (2004) 215-226.

8. Frehaut, J., NIM, 135, (1976) 511-518.

9. H. Beer and F. Käppeler, Phys. Rev C, 21, (1980) 534-544.

10. H. J. Brede et al, NIM, 169, (1980) 349-358.

11. P. W. Lisowski, et al, Nuc. Sci. Eng., 106, (1990) 208.

12. M. J. Burns, et al, Proc. Of 1999 Particle Acc. Conf., (1999) 617-621.

13. R. O. Bangerter, NIMA, 464, (2001) 17-23.

14. R. Reifarth, et al, ApJ, 581, (2003) 1251. 